\title{
Amelioration of 1, 2 Dimethylhydrazine Induced Tumor Promotion Response by Novel Benzimidazole Derivative Nanoparticle in Wistar Rats
}

\author{
S.S. Rajendran ${ }^{1 *}$, G. Geetha ${ }^{2}$, R. Venkatanarayanan ${ }^{1}$, N. Santhi ${ }^{1}$ \\ 'Department of Pharmaceutical Chemistry, RVS College of Pharmaceutical Sciences, Sulur, Coimbatore, Tamil Nadu - 641 402, INDIA. \\ 2Department of Pharmaceutical Chemistry, PPG College of Pharmacy, Saravanampatti, Coimbatore, Tamilnadu-641 035, INDIA.
}

\begin{abstract}
Background: Colon cancer is one of the most common malignancies in many regions of the world and is thought to arise from the accumulation of mutations in a single epithelial cell of the colon and rectum. The benzimidazole comprises a important pharmacophore and privileged structure in modern drug discovery. Various substituted benzimidazole derivatives have been found to possess potential anticancer properties. Objective: The study aimed to prove the anti-colon cancer activity of novel benzimidazole derivative 4-(1H-benzo[d]imidazol-2-yl)-6-phenylpyrimidin-2-amine loaded chitosan nanoparticle (BZI 3 nano) by an 1, 2 Dimethylhydrazine (DMH) Induced rat model in-vivo study and identify the targeting efficiency of BZI 3 nano to treat colorectal cancer. Method: The effect of novel benzimidazole derivative 4-(1H-benzo[d]imidazol-2-yl)-6-phenylpyrimidin-2-amine loaded chitosan nanoparticle (BZI 3 nano) on the formation of aberrant crypt foci (ACF), apoptosis, histopathology, body weight, organs weight and heamotological parameters were studied in 1,2-dimethylhydrazine (DMH)-induced colon cancer in rats. Results: BZI 3 nano $(5 \mathrm{mg} / \mathrm{kg}, p . o)$ administration
\end{abstract}

significantly reduced ACF number and increased the weight gain and apoptotic index compared to DMH treated group. The histological alterations induced by DMH were also significantly improved. Conclusion: In-vivo anticancer activities results revealed that the presence substituted benzimidazole derivative nanoparticle (BZI 3 nano) could have the anticancer potential of the scaffold and selective, good target for drug discovery, which can be regarded as promising anticancer potential.

Key words: Benzimidazole derivative, Nanoparticles, DMH, ACF.

Correspondence

S.S. Rajendran, Assistant Professor, Department of Pharmaceutical Chemistry, RVS College of Pharmaceutical Sciences, Coimbatore, Tamil Nadu - 641402, INDIA.

Phone: +919894897258

Email: researchrajendran@gmail.com

DOI: 10.5530/jyp.2018.10.65

\section{INTRODUCTION}

Cancers are the second cause of death and have the highest burden of diseases in $21^{\text {st }}$ century. The annual cancer deaths will increase from 7.6 million in 2008 to 13 million in 2030, according to the WHO world health statistics report 2012. For the survival of any organism, there should be a delicate balance between the cell growth and death. This balance can get disturbed in a number of ways, which may lead to either abnormal growth of tissue or may develops into a lethal tumor or cancer. Cancer is a major public health burden in both developed and developing countries. Anticancer activity is the effect of natural and synthetic or biological and chemical agents to reverse, suppress or prevent carcinogenic progression. ${ }^{1}$ Colon cancer is the second leading cause of cancer related deaths in USA. ${ }^{2}$ Recently colon cancer incidence has been increasing in India year by year. ${ }^{3}$ The efficacy of present cancer chemotherapy is mainly limited by the toxicity associated with the anticancer drugs to normal tissues. This limitations result from the fact that anticancer drugs presently used in chemotherapy lack efficient selectivity towards tumor cells. This necessitates the development of a novel nanoparticle delivery system to overcome these current obstacles in convention drug therapy. ${ }^{4}$ Benzimidazole derivatives are comprises a relatively huge, growing, key pharmacophore and privileged structure in modern drug discovery. ${ }^{5}$ Now a days is a moiety of choice which has numerous pharmacological properties. Substituted benzimidazole derivatives have found applications in diverse therapeutical areas. ${ }^{6}$ Principally 2 -substituted benzimidazole derivatives have been found to possess potential anticancer properties. ${ }^{7}$
A rational approach for design the next generation inhibitors, benzimidazole derivatives, ${ }^{8}$ The novel benzimidazole derivative 4 - $(1 \mathrm{H}$-benzo[d] imidazol-2-yl)-6-phenylpyrimidin-2-amine loaded chitosan nanoparticles formulated and characterized, these results demonstrate that the possibility of delivering synthesised novel benzimidazole derivative 4-(1H-benzo[d]imidazol-2-yl)-6-phenylpyrimidin-2-amine loaded nanoparticle (BZI 3 nano) to colorectum with enhanced encapsulation efficiency. Additionally the nanoparticle (BZI 3 nano) have been evaluated for In vitro cytotoxicity in Caco 2 and MCF- 7 cell lines. In vitro cytotoxicity study suggested the safety of the prepared novel nanoparticle (BZI 3 nano), which can be potential carrier to deliver hydrophilic drugs to target colorectum. ${ }^{9}$ Further In vivo will confirm the targeting efficiency of nanoparticle (BZI 3 nano) to treat colorectal cancer.

Hence the present work was undertaken to scientifically prove the anticolon cancer activity of novel benzimidazole derivative 4-(1H-benzo[d] imidazol-2-yl)-6-phenylpyrimidin-2-amine loaded chitosan nanoparticle (BZI 3 nano) by an in-vivo study.

\section{MATERIALS AND METHODS}

Reagents and Chemicals

1, 2-Dimethyl hydrazine (DMH) were purchased from Sigma Chemicals private limited, Bangalore, Methylene blue from Sisco Research Laboratories private limited, Mumbai, Haematoxylin from Merck Specialties private limited, Mumbai. All other chemicals and reagents used were of analytical grade. 


\section{Drug Profile $e^{9-10}$}

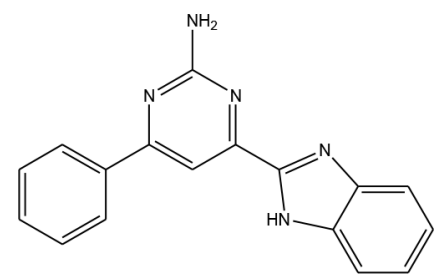

4-(1H-benzo[d]imidazol-2-yl)-6-phenylpyrimidin-2amine

Molecular Formula: $\mathrm{C}_{17} \mathrm{H}_{13} \mathrm{~N}_{5}$ : Molecular Weight: 287.32

Mp: $146-149{ }^{\circ} \mathrm{C}: \mathrm{R} f: 0.75$

The 2- substituted novel benzimidazole derivative were synthesised by using incorporated with heterocycle like pyrimidine and backbone of chalcones. The synthesised compound were screened for their in vitro anticancer activities against the CaCo-2 and MCF-7 cell lines. The 24 hours Caspase study revealed that compound $4-(1 \mathrm{H}-$ benzo[d]imidazol2-yl)-6-phenylpyrimidin-2-amine showed 2 fold activities in caspase 3 and 9 pathway, single fold activity in caspase 8 pathways. The novel benzimidazole derivative 4-(1H-benzo[d]imidazol-2-yl)-6-phenylpyrimidin2 -amine loaded chitosan nanoparticles formulated and characterized. These results revealed that the presence 2 -substituted benzimidazole derivative could have the anticancer potential of the scaffold.

\section{Animals ${ }^{11-13}$}

In this study, A total of 24 Male Wistar albino rats (150-180 gm) were selected and procured from RVS College Of Pharmaceutical Sciences, Tamilnadu, India. All animals were maintained under environmentally controlled conditions of $25 \pm 2^{\circ} \mathrm{C}$, relative humidity of 45 to $55 \%$ and $12 \mathrm{~h}$ light- $12 \mathrm{~h}$ dark cycle. The animals were acclimatized to laboratory conditions at least one week before starting the experiment and they had free access to food and water ad labitum. The study protocol was approved by Institutional Animal Ethics Committee (IAE1012/c/16/ CPCSEA - Corres - 2013).

Acute Toxicity Study ${ }^{14-15}$

Acute toxicity studies and fixing of dose for the newly formulated benzimidazole derivative nanoparticle (BZI 3 nano). Acute oral toxicity test was carried out according to the OECD guideline No. 423.Male Wistar Albino Rats were kept for overnight fasting prior to drug administration. The sighting study of the compound was carried out on the group (3 numbers) of albino rats of wistar strain of male sex fixed dose method. The animals were observed for a period of $24 \mathrm{hrs}$ for the changes in behaviour, hypersensitivity reactions etc. Mortality, if any, was determined over a period of 2 weeks.

\section{Preparation of $\mathrm{DMH}$}

DMH was dissolved in $1 \mathrm{mM}$ EDTA just prior to use and the $\mathrm{pH}$ adjusted to 6.5 with $1 \mathrm{mM} \mathrm{NaOH}$ to ensure the stability of the carcinogen.

\section{Induction of colon cancer}

The rats were given subcutaneous injections of DMH twice a week for 2 weeks at a dose of $30 \mathrm{mg} / \mathrm{kg}$ body weight.

Preparation of standard drug (5-Fluorouracil)

The 5-Fluorouracil was dissolved in normal saline

\section{Preparation of test drug (BZI 3 nano)}

BZI 3 nano was suspended in 5\% gum acacia and each rat received a daily $1 \mathrm{ml}$ as suspension at a dose of $5 \mathrm{mg} / \mathrm{kg}$ body weight orally by oral gavage throughout the experimental period.

\section{Experimental Design and Treatment Schedule ${ }^{16}$}

Group I served as normal control, which received vehicle (gum acacia suspension), Group II rats served as disease control were given subcutaneous injections of DMH twice a week for 2 consecutive weeks at $30 \mathrm{mg} / \mathrm{kg}$ b.w., in addition to this Group III rats were given standard (5-Fluorouracil) at a dose of $20 \mathrm{mg} / \mathrm{kg}$ body weight daily by i.p and Group IV rats were given formulated novel benzimidazole derivative nanoparticle (BZI 3 nano) at a dose of $5 \mathrm{mg} / \mathrm{kg}$ body weight daily for total 30 days of study period by oral route.

Group I : Vehicle control: received $1 \mathrm{ml}$ of normal saline (p.o)

Group II : Disease control (DMH: $30 \mathrm{mg} / \mathrm{Kg}$, s.c)

Group III: Disease + 5-Fluorouracil (5-FU: $20 \mathrm{mg} / \mathrm{kg}$, i.p)

Group IV: Disease + BZI 3 nano $(5 \mathrm{mg} / \mathrm{kg}$, p.o $)$

In vivo Methods

Body Weight Changes ${ }^{17-18}$

The body weight changes of the Control, DMH, 5-FU and BZI 3 nano treated rats were measured throughout the study. The rats were weighed at the beginning of the experiment and then subsequently once a week and final before sacrifice.

\section{Determination of Aberrant Crypt Foci (ACF) ${ }^{16}$}

At the end of the study, rat colons were removed and flushed with Potassium Phosphate buffered saline (0.1M, pH 7.2) and colons were split open longitudinally and placed on strips of filter paper with their luminal surfaces open and exposed. Another strip of filter paper was placed on top of the luminal surface. The colons were then secured and fixed in a tray containing $10 \%$ buffered formalin for overnight. Each of the fixed colons was cut into proximal and distal portions of equal lengths and portion was further cut into $2 \mathrm{~cm}$ long segments.

Each segment was placed in a Petri dish and stained with $0.2 \%$ methylene blue solution for $2 \mathrm{~min}$. The segment was examined using a light microscope at low magnification to score the total number of ACF as well as the number of crypts per focus. ACF were distinguished from normal crypts by their thicker, darker-stained, raised walls with elongated slit-like lumens and significantly increased distance from the lamina to basal surface off cells.

\section{Apoptosis Measurement in Colonic Mucosa ${ }^{19}$}

Apoptosis evaluation was carried out in paraffin-embedded section of normal colonic mucosa and tumours stained with haematoxylin. At least 20 full longitudinal crypt sections of normal mucosa of rat were scored at the microscope, determainining the presence of cells in each crypt with the following characteristics of apoptosis: cell shrinkage, loss of normal contact with the adjacent cell, chromatin condensation or formation of round or oval nuclear fragments. When clusters of more than one apoptotic fragments. When clusters of more than one apoptotic body were seen within the diameter of one cell, these bodies were considered as fragments of one apoptotic cell. Tumour apoptosis was determined by scoring at least $1000 \mathrm{cells} / \mathrm{rat}$ for the presence of apoptotic cells that were coded as described. In tumours and colon mucosa, apoptosis was scored by a single observer on coded samples and quantified as apoptotic index (AI).

$$
A I=\text { number of apoptotic cells/cells scored } \times 100 \text {. }
$$

\section{Haematological Evaluation ${ }^{20}$}

Before the initiation of the study and immediately before necropsy, blood samples were collected for haematological analysis in EDTA tubes with 1.5\%EDTA and differentially quantified through a coulter T890 for the following: leukocyte, erythrocyte, platelet counts and haemoglobin determination. 


\section{Individual Organs Weights ${ }^{20}$}

At the end of the study animals were sacrified and remove the all visceral organs like liver, kidney, colon, spleen, heart, pancreas, stomach, lungs were removed, weighed and relative weight of organs were calculated.

\section{Histopathology of Colon ${ }^{21}$}

The colon were excised, flushed with saline, cut open longitudinally along the main axis and then again washed with saline. These colonic sections fixed in $10 \%$ buffered formalin for at least $24 \mathrm{~h}$ and after fixation, the specimens were dehydrated in ascending grades of ethanol, cleared in benzene and embedded in paraffin wax. Blocks were made and $5 \mu \mathrm{m}$ thick sections were cut from the distal colon. The paraffin embedded colonic tissue sections were deparaffinised using xylene and ethanol. These sections stained with haematoxylin and eosin and were observed under light microscope at 10X and 40X magnifications to investigate the histo architecture of colonic mucosa.

\section{Statistical Analysis}

Results were given as means \pm SD of each group. Data were analyzed by one-way analysis of variance and any significant difference among treatment groups was evaluated by Dunnet's Test. The results were considered statistically significant at $\mathrm{P}<0.05$.

\section{RESULTS}

\section{Acute Toxicity Study}

Acute toxicity studies and fixing of dose for the newlyl formulated benzimidazole derivative nanoparticle (BZI 3 nano). Acute oral toxicity test was carried out according to the OECD guideline No. 423.Male Wistar Albino Rats were kept for overnight fasting prior to drug administration. The sighting study of the compound was carried out on the group (3 numbers) of albino rats of wistar strain of male sex following fixed dose method. Only one animal among the group of three was tested with initial dose $5 \mathrm{mg} / \mathrm{kg}$ via orally administration and observe once in $30 \mathrm{~min}$ for the first $4 \mathrm{~h}$ and periodically during the first $24 \mathrm{~h}$ for event toxicity or mortality. Since there was no such toxicity or mortality, the other animals in the group were testes with initial test dose $5 \mathrm{mg} / \mathrm{kg}$ and observed for 14 days, the test compound did not produce any signs of toxicity or mortality. The procedure of sighting study was repeated at a higher dose of $25 \mathrm{mg} / \mathrm{kg}$ and observed for 14 days, and the test compounds did not produce any signs of toxicity or mortality. At $50 \mathrm{mg} / \mathrm{kg}$ sighting study, the compound produced mortality in more than $40 \%$ of the population with tremors and convulsion. Hence, $5 \mathrm{mg} / \mathrm{kg}$ was fixed the dose strength for the main study.

\section{In vivo Methods}

\section{Body Weight Changes}

During the experimental period 30 days, the carcinogen-exposed rats (Group II- DMH treated) exhibited a significantly $(p<0.05)$ low gain in body weight (Figure 1) and a low growth rate throughout the experimental period as compared to Groups III (DMH+5FU) and IV (DMH+ BZI 3 nano) Oral administration of BZI 3 nano at a dose of $5 \mathrm{mg} / \mathrm{kg}$ b.w resulted in a significant improvement in weight gain relative to treatment with DMH alone. (Table 1)

\section{Determination of Aberrant Crypt Foci (ACF)}

$\mathrm{ACF}$ formation was observed in all $\mathrm{DMH}$ induced groups. The majority of ACF appeared in the distal colon of the rats injected with DMH. Oral administration of BZI 3 nano at $5 \mathrm{mg} / \mathrm{kg}$ b.w. inhibited the formation as well as the total number of ACF (Table 2 and Figure 2), as compared to rats injected with $\mathrm{DMH}$ alone. No ACF formation was observed in the control (group I - Control).
Table 1: Body weight changes on treatment with $\mathrm{DMH}, 5 \mathrm{FU}$ and BZI 3 nano.

\begin{tabular}{cccc}
\hline Group/Treatment & $\begin{array}{c}\text { Initial body } \\
\text { weight(g) }\end{array}$ & $\begin{array}{c}\text { Final body } \\
\text { weight(g) }\end{array}$ & Weight gain(g) \\
\hline Control & $165.16 \pm 8.93$ & $216.50 \pm 7.04$ & $51.34 \pm 7.92$ \\
DMH & $162.50 \pm 7.72$ & $191.83 \pm 8.72$ & $29.33 \pm 5.24$ \\
DMH+5FU & $173.33 \pm 7.93$ & $217.60 \pm 6.54$ & $44.27 \pm 3.04^{* * *}$ \\
DMH+ BZI 3 nano & $163.50 \pm 6.54$ & $203.33 \pm 6.70$ & $39.83 \pm 4.544^{* *}$ \\
\hline
\end{tabular}

Values are mean $\pm \mathrm{SD}, \mathrm{n}=6$ in each group, statistically significant ${ }^{* * *} \mathrm{p}<0.001$ ${ }^{* *} \mathrm{p}<0.01,{ }^{*} \mathrm{p}<0.05$ when compared with disease control (DMH treated) group (One way ANOVA followed by Dunnet's test).

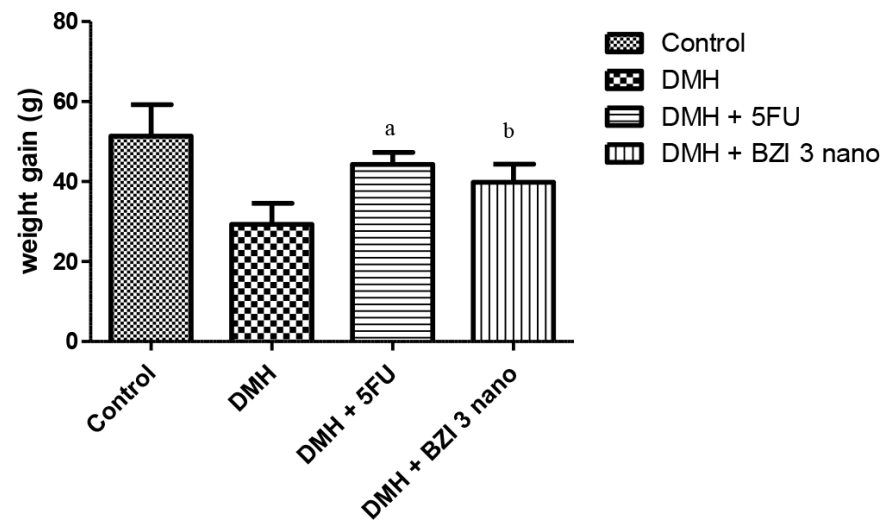

Figure 1: Body weight changes on treatment with $\mathrm{DMH}, 5 \mathrm{FU}$ and $\mathrm{BZI} 3$ nano. Values are mean $\pm S D, n=6$ in each group, statistically significant $a^{* * *} p<0.001$, $b * 0<0.01$ when compared with disease control (DMH treated) group (One way ANOVA followed by Dunnet's test).

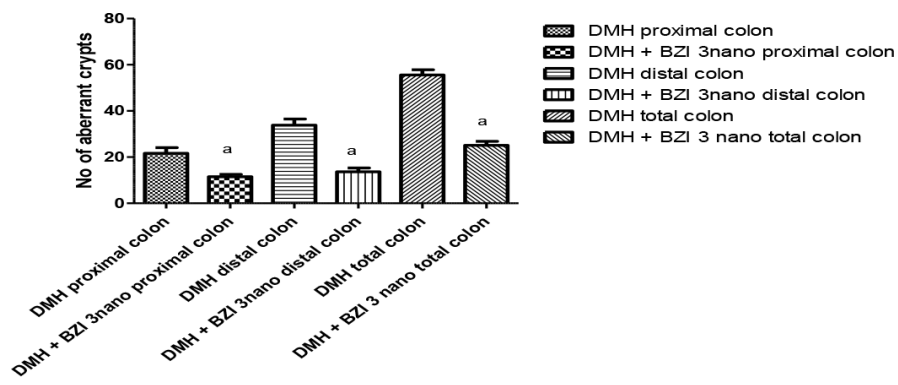

Figure 2: Aberrant crypt foci scoring

Values are mean $\pm S D, n=6$ in each group, statistically significant $a^{* * *} p<0.001$, when compared with disease control (DMH treated) group (One way ANOVA followed by Dunnet's test).

Regional distribution of aberrant crypt categories (1, 2, 3, 4 and $\geq 5)$ in rats treated with $D M H, 5 F U$ and $B Z I 3$ nano.

ACF formation was observed in all DMH induced groups. The majority of ACF appeared in the distal colon of the rats injected with DMH. Oral administration of BZI 3 nano at $5 \mathrm{mg} / \mathrm{kg}$ b.w. inhibited the formation as well as the total number of ACF, AC (Table 3 and Figure 3 ) as compared to rats injected with DMH alone. No ACF formation was observed in the control (Group I -control).

\section{Apoptosis Measurement in Colonic Mucosa}

The apoptosis index (AI \%) was estimated as the percentage of apoptotic cells (i.e., with cellular retraction and condensation, condensed or fragmented nuclear chromatin and formation of apoptotic bodies) among 
Table 2: Aberrant crypt foci scoring - Distribution of altered aberrant crypt foci (ACF) category in proximal, distal and total colon of rats exposed to DMH, 5FU and BZI 3 nano.

\begin{tabular}{|c|c|c|c|c|}
\hline Group/Treatment & No. of animals & Total no. of AC & Total no. of ACF & Crypt/ACF \\
\hline \multicolumn{5}{|c|}{ Proximal colon } \\
\hline Control & 6 & 0 & 0 & 0 \\
\hline $\mathrm{DMH}$ & 6 & $21.66 \pm 2.47$ & $16.16 \pm 3.41$ & $1.34 \pm 0.70$ \\
\hline $\mathrm{DMH}+5 \mathrm{FU}$ & 6 & $15.33 \pm 3.26$ & $10.50 \pm 1.19$ & $1.46 \pm 0.81$ \\
\hline $\mathrm{DMH}+\mathrm{BZI} 3$ nano & 6 & $11.50 \pm 1.02$ & $9.33 \pm 1.77$ & $1.23 \pm 0.83$ \\
\hline \multicolumn{5}{|c|}{ Distal colon } \\
\hline Control & 6 & 0 & 0 & 0 \\
\hline $\mathrm{DMH}$ & 6 & $33.83 \pm 2.68$ & $19.16 \pm 2.66$ & $1.76 \pm 0.51$ \\
\hline $\mathrm{DMH}+5 \mathrm{FU}$ & 6 & $24.16 \pm 1.68$ & $15.66 \pm 3.27$ & $1.54 \pm 0.40$ \\
\hline $\mathrm{DMH}+\mathrm{BZI} 3$ nano & 6 & $13.60 \pm 1.72$ & $9.50 \pm 1.34$ & $1.43 \pm 0.72$ \\
\hline \multicolumn{5}{|c|}{ Total colon } \\
\hline Contro & 6 & 0 & 0 & 0 \\
\hline $\mathrm{DMH}$ & 6 & $55.59 \pm 2.25$ & $35.32 \pm 1.64$ & $1.57 \pm 0.83$ \\
\hline $\mathrm{DMH}+5 \mathrm{FU}$ & 6 & $39.49 \pm 1.47$ & $26.16 \pm 1.21$ & $1.50 \pm 0.81$ \\
\hline DMH+ BZI 3 nano & 6 & $25.10 \pm 1.75^{* * *}$ & $18.83 \pm 1.64^{\star * \star}$ & $1.33 \pm 0.80^{* * *}$ \\
\hline
\end{tabular}

Values are mean $\pm S D, n=6$ in each group, statistically significan ${ }^{* * *} \mathrm{p}<0.001{ }^{* *} \mathrm{p}<0.01,{ }^{*} \mathrm{p}<0.05$ when compared with disease control (DMH treated) group (One way ANOVA followed by Dunnet's test).

Table 3: Regional distribution of aberrant crypt categories (1, 2, 3, 4 and $\geq 5)$ in rats treated with DMH, 5FU and BZI 3 nano.

\begin{tabular}{|c|c|c|c|c|c|c|}
\hline \multirow[t]{2}{*}{ Group/Treatment } & \multirow[t]{2}{*}{ No. of animals } & \multicolumn{5}{|c|}{ Number of aberrant crypts per ACF } \\
\hline & & 1 & 2 & 3 & 4 & $\geq 5$ \\
\hline \multicolumn{7}{|c|}{ Proximal colon } \\
\hline Control & 6 & 0 & 0 & 0 & 0 & 0 \\
\hline $\mathrm{DMH}$ & 6 & $8.33 \pm 3.61$ & $6.83 \pm 2.26$ & $3.16 \pm 1.72$ & $2.50 \pm 1.86$ & $2.33 \pm 1.37$ \\
\hline $\mathrm{DMH}+5 \mathrm{FU}$ & 6 & $5.83 \pm 2.33$ & $4.33 \pm 3.17$ & $2.83 \pm 1.68$ & $1.83 \pm 1.23$ & $1.66 \pm 0.52$ \\
\hline $\mathrm{DMH}+\mathrm{BZI} 3$ nano & 6 & $3.83 \pm 1.67^{* *}$ & $2.83 \pm 0.17$ & $2.16 \pm 1.32$ & $1.33 \pm 0.87$ & $1.16 \pm 0.89$ \\
\hline \multicolumn{7}{|c|}{ Distal colon } \\
\hline Control & 6 & 0 & 0 & 0 & 0 & 0 \\
\hline $\mathrm{DMH}$ & 6 & $15.66 \pm 3.77$ & $16.50 \pm 5.62$ & $12.16 \pm 1.06$ & $10.83 \pm 1.67$ & $8.16 \pm 0.63$ \\
\hline $\mathrm{DMH}+5 \mathrm{FU}$ & 6 & $10.16 \pm 4.27$ & $14.33 \pm 3.67$ & $8.50 \pm 4.4$ & $8.33 \pm 2.81$ & $7.33 \pm 4.5$ \\
\hline $\mathrm{DMH}+\mathrm{BZI} 3$ nano & 6 & $8.16 \pm 2.82^{* *}$ & $10.16 \pm 2.12^{\star *}$ & $5.16 \pm 2.89$ & $5.33 \pm 2.16$ & $5.16 \pm 1.80^{*}$ \\
\hline \multicolumn{7}{|c|}{ Total colon } \\
\hline Control & 6 & 0 & 0 & 0 & 0 & 0 \\
\hline $\mathrm{DMH}$ & 6 & $23.99 \pm 1.82$ & $23.33 \pm 1.34$ & $15.32 \pm 1.38$ & $13.33 \pm 3.20$ & $10.49 \pm 2.04$ \\
\hline $\mathrm{DMH}+5 \mathrm{FU}$ & 6 & $15.99 \pm 2.06$ & $18.66 \pm 2.16$ & $11.33 \pm 2.85$ & $10.16 \pm 2.16$ & $8.99 \pm 1.67$ \\
\hline $\mathrm{DMH}+\mathrm{BZI} 3$ nano & 6 & $11.99 \pm 2.50^{* *}$ & $12.99 \pm 2.80^{\star *}$ & $7.32 \pm 1.90$ & $6.66 \pm 1.64$ & $6.32 \pm 2.07$ \\
\hline
\end{tabular}

Values are mean $\pm S D, n=6$ in each group, statistically significant ${ }^{* *} \mathrm{p}<0.01, \mathrm{~b}^{*} \mathrm{p}<0.05$ when compared with disease control (DMH treated) group (One way ANOVA followed by Dunnet's test).

the total number of counted cells in a whole colonic crypt. The apoptotic index was increased in group treated with DMH+ BZI 3 nano compared to $\mathrm{DMH}$ treated group and $\mathrm{DMH}+5 \mathrm{FU}$ group. (Table 4 and Figure 4 ).

\section{Haematological Evaluation}

Various haematological parameters are compared among the groups before and after induction of colon cancer as shown in Table 5.
Individual Organs Weights

Relative weights of various organs are compared among the groups after induction of colon cancer as shown in Table 6.

\section{Histopathology of Colon}

Pathology of haematoxylin and eosin stained sections of colon slides showed that there was a wide range of histology from minor atypia to 


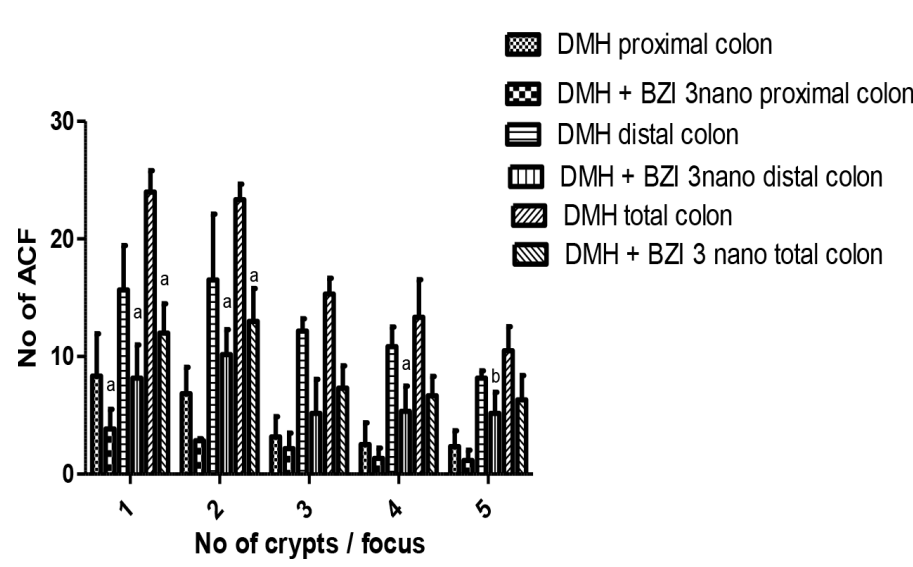

Figure 3: Regional distribution of aberrant crypt categories

severe dysplasia in DMH treated group than BZI 3 nano treated group. There was no change in colonic mucosa of control group rats (Figure 5).

\section{DISCUSSION}

In an effort to establish novel benzimidazole derivative with anticancer activity and achieve the colon target, we performed In vivo method to confirm the targeting efficiency of synthesised novel benzimidazole derivative 4-(1H-benzo[d]imidazol-2-yl)-6-phenylpyrimidin-2-amine loaded chitosan nanoparticle (BZI 3 nano) to treat colorectal cancer.

Acute toxicity studies performed on newly formulated benzimidazole derivative nanoparticle (BZI 3 nano) (according to OECD guidelines 423). At $50 \mathrm{mg} / \mathrm{kg}$ sighting study, the compound produced mortality in more than $40 \%$ of the population with tremors and convulsion. Hence, from this 1/10th of MTD was selected and the effective doses were fixed as $5 \mathrm{mg} / \mathrm{kg}$ dose strength for the further pharmacological studies. ${ }^{15}$

In vivo study we used $\mathrm{DMH}$ as colon cancer inducer and synthesized novel benzimidazole derivative nano particle (BZI 3 nano) as the test drug.

Weight loss reflects a common feature of gastrointestinal tumours, and weight loss is a frequent cause of concern, the aggressiveness of the disease. As expected carcinogen (DMH) treated animals in our study showed decreased weight gain compared to other groups. It may be due to reduced food intake. In addition to increased hepatic gluconeogenesis and altered glucose metabolism reduces the energy sources leading to a significant weight loss in DMH treated animals. ${ }^{18}$

\begin{tabular}{cc} 
Table 4: Effect of DMH, $\mathbf{5 F U}$ and BZI $\mathbf{3}$ nano on apoptotic indexes. \\
\hline Group/Treatment & Apoptotic index \\
\hline Control & 4.16 \\
DMH & 0.80 \\
DMH+5FU & $3.33^{* *}$ \\
DMH+ BZI 3 nano & $3.83^{* *}$ \\
\hline
\end{tabular}

Values are mean $\pm S D, n=6$ in each group, statistically significant ${ }^{* *} \mathrm{p}<0.01$ when compared with disease control (DMH treated) group (One way ANOVA followed by Dunnet's test).

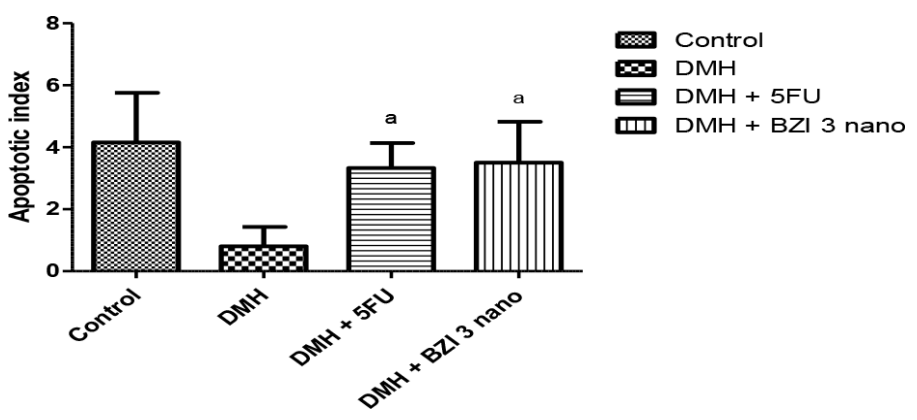

Figure 4: Effect of DMH, 5FU and BZI 3 nano on apoptotic indexes. Values are mean $\pm S D, n=6$ in each group, statistically significant $a^{* *} p<0.01$, when compared with disease control (DMH treated) group (One way ANOVA followed by Dunnet's test).

In this study, colons were examined for ACF 30days after the first injection of DMH. In our present study increased crypt number was observed in proximal, distal and total colons of DMH-treated rats than in treatment group. We also identified that the inhibitory effect of BZI 3 nano on the development of ACF was more pronounced in the entire period treatment regimen as compared to the other treatment groups. Data from ACF incidence indicated that all rats treated with DMH developed ACF. The results demonstrated that administration of BZI 3 nano suppress DMH-induced ACF development. BZI 3 nano did not affect the number of crypts per ACF but actually suppressed the total number of ACF. Besides, the decrease in numbers of ACF suggests that BZI 3 nano may inhibit the growth of ACF. The no of ACF in the distal colon were more than the proximal colon. ${ }^{19}$

Table 5: Effect of DMH, 5FU and BZI 3 nano on various haematological parameters.

\begin{tabular}{|c|c|c|c|c|c|c|c|c|}
\hline \multirow{3}{*}{$\begin{array}{l}\text { Hemotological } \\
\text { parameters }\end{array}$} & \multicolumn{8}{|c|}{ Change in hemotological parameters after induction } \\
\hline & \multicolumn{2}{|c|}{ Control } & \multicolumn{2}{|c|}{$\mathrm{DMH}$} & \multicolumn{2}{|c|}{$\mathrm{DMH}+5 \mathrm{FU}$} & \multicolumn{2}{|c|}{$\mathrm{DMH}+\mathrm{BZI} 3$ nano } \\
\hline & Before & After & Before & After & Before & After & Before & After \\
\hline $\begin{array}{c}\text { WBC } \\
\left(\text { cells } / \mu \mathrm{L} \times 10^{3}\right)\end{array}$ & $11.16 \pm 1.8$ & $11.33 \pm 2.4$ & $11.50 \pm 1.90$ & $14.83 \pm 2.40$ & $11.83 \pm 2.47$ & $8.66 \pm 3.50^{* *}$ & $11.66 \pm 3.45$ & $9.50 \pm 4.20^{* *}$ \\
\hline $\begin{array}{c}\mathrm{RBC} \\
\left(\text { cells } / \mu \mathrm{L} \times 10^{6}\right)\end{array}$ & $6.83 \pm 0.06$ & $7.50 \pm 0.26$ & $6.50 \pm 1.09$ & $5.66 \pm 1.20$ & $6.16 \pm 1.40$ & $4.66 \pm 0.80^{* *}$ & $6.50 \pm 1.05$ & $4.83 \pm 0.0^{* *}$ \\
\hline $\mathrm{HGB}(\mathrm{g} / \mathrm{dL})$ & $11.83 \pm 1.08$ & $12.16 \pm 1.04$ & $11.33 \pm 2.32$ & $9.50 \pm 2.56$ & $11.83 \pm 2.02$ & $7.50 \pm 1.08^{*}$ & $11.16 \pm 2.50$ & $9.83 \pm 1.40^{* *}$ \\
\hline $\begin{array}{c}\text { PLT } \\
\left(\text { cells } / \mu \mathrm{L} \times 10^{3}\right)\end{array}$ & $185.83 \pm 18.78$ & $187.33 \pm 19.06$ & $185.50 \pm 15.64$ & $172.16 \pm 1.45$ & $185.16 \pm 15.45$ & $167.66 \pm 16.50$ & $185.83 \pm 16.21$ & $170.16 \pm 15.40$ \\
\hline HCT (\%) & $30.16 \pm 1.21$ & $32.50 \pm 4.09$ & $30.66 \pm 3.50$ & $27.83 \pm 4.65$ & $30.50 \pm 5.80$ & $22.50 \pm 4.20$ & $30.83 \pm 5.40$ & $28.66 \pm 4.65$ \\
\hline
\end{tabular}

Values are Mean \pm S.E.M, $\mathrm{n}=6$ in each group. ${ }^{\star} P<0.05,{ }^{* *} P<0.01,{ }^{* * *} P<0.001$ when compared with disease control group (One way ANOVA followed by Dunnett's test). 
Table 6: Effect of DMH, 5FU and BZI 3 nano on relative weight of organs.

\begin{tabular}{|c|c|c|c|c|}
\hline \multirow{2}{*}{$\begin{array}{l}\text { Relative wt of organs } \\
\text { (wt of organ/100g) }\end{array}$} & \multicolumn{4}{|c|}{ Group/Treatment } \\
\hline & Control & $\mathrm{DMH}$ & $\mathrm{DMH}+5 \mathrm{FU}$ & $\mathrm{DMH}+\mathrm{BZI} 3$ nano \\
\hline Liver & $16.85 \pm 0.07$ & $12.29 \pm 0.4$ & $14.61 \pm 0.06^{* *}$ & $13.50 \pm 0.18^{\star \star}$ \\
\hline Kidneys & $0.93 \pm 0.04$ & $0.52 \pm 0.02$ & $0.7 \pm 0.01^{* *}$ & $0.66 \pm 0.02^{\star *}$ \\
\hline Heart & $0.49 \pm 0.03$ & $0.23 \pm 0.02$ & $0.39 \pm 0.01$ & $0.32 \pm 0.01$ \\
\hline Lungs & $0.85 \pm 0.01$ & $0.51 \pm 0.01$ & $0.74 \pm 0.01^{\star *}$ & $0.69 \pm 0.01^{* * *}$ \\
\hline Pancreas & $0.81 \pm 0.02$ & $0.57 \pm 0.01$ & $0.78 \pm 0.005$ & $0.71 \pm 0.02$ \\
\hline Spleen & $0.39 \pm 0.01$ & $0.19 \pm 0.01$ & $0.35 \pm 0.01^{* *}$ & $0.31 \pm 0.01^{* * *}$ \\
\hline Colon & $0.78 \pm 0.01$ & $0.56 \pm 0.02$ & $0.7 \pm 0.01$ & $0.64 \pm 0.05$ \\
\hline Stomach & $0.80 \pm 0.01$ & $0.48 \pm 0.01$ & $0.72 \pm 0.01$ & $0.65 \pm 0.03$ \\
\hline
\end{tabular}

Values are Mean \pm S.E.M, $\mathrm{n}=6$ in each group. ${ }^{\star} \mathrm{P}<0.05,{ }^{* *} \mathrm{P}<0.01,{ }^{* * *} \mathrm{P}<0.001$ when compared with disease control group (One way ANOVA followed by Dunnett's test).
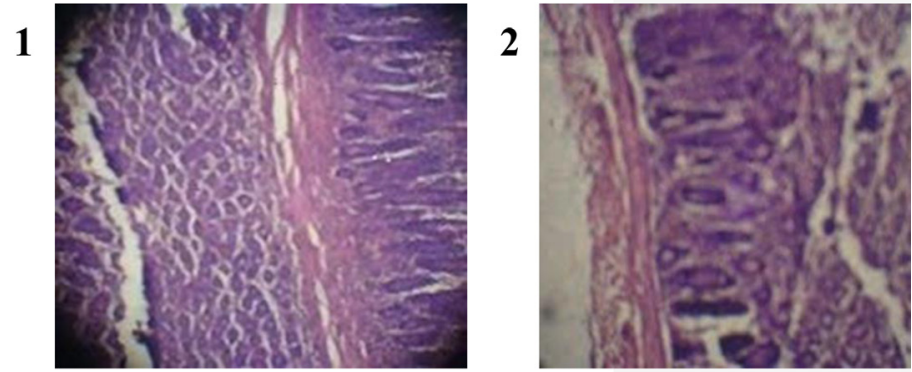

3
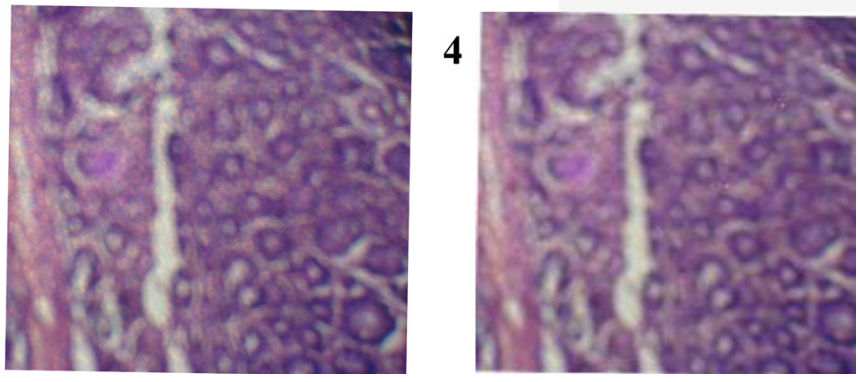

Figure 5: Histopathology of Colon.

(1) Control, (2) DMH Treated, (3) DMH + 5 FU, (4) DMH + BZI 3 nano

(1) Topographical view of normal crypt (40X). (2) Histological changes

in the colonic mucosa on DMH administration shows thickened mucosa with densely packed inflammatory cell infiltration and a higher degree of hyperplasia (40x). (3) Represents $20 \mathrm{mg} / \mathrm{kg}$ b.w., $5 \mathrm{FU}$ supplemented rat colon showing mucosal thickening and scattered or no infiltration of inflammatory cells in the mucosal layer (40X). (4) Represents $5 \mathrm{mg} / \mathrm{kg}$ b.w., BZI 3 nano supplemented rat colon showing mucosal thickening in few areas and scattered or no infiltration of inflammatory cells in the mucosal layer (40x).

Pathology of haematoxylin and eosin stained sections of colon slides revealed that there was a broad range of histology from minor atypia to severe dysplasia in DMH treated group than BZI 3 nano treated group. There was no change in colonic mucosa of control group rats. ${ }^{20}$

Besides the reduction of ACF, based on the results it also revealed that BZI 3 nano induces apoptosis the apoptotic index was high in rats treated with $\mathrm{DMH}$ alone.

The results of the present study showed that administration of BZI 3 nano at a dose of $5 \mathrm{mg} / \mathrm{kg}$ body weight during either the initiation, postinitiation or entire period phase significantly inhibited $\mathrm{DMH}$-induced colon carcinogenesis in rats. Our findings indicate that BZI 3 nano is significantly reduced the number of ACF development in the colon.
Several studies have suggested the growth features of ACF and dysplastic $\mathrm{ACF}$ and their location as a measure of the biological efficacy of the modifiers of colon carcinogenesis. Thus the data strongly suggest that the colon cancer inhibitory effects observed with BZI 3 nano at a dose of $5 \mathrm{mg} / \mathrm{kg}$ body weight. The inhibitory effect of BZI 3 nano may also due to the induction of apoptosis.

\section{CONCLUSION}

In vivo cytotoxicity study suggested the safety of the prepared synthesized novel benzimidazole derivative 4-(1H-benzo[d]imidazol-2-yl)6-phenylpyrimidin-2-amine loaded chitosan nanoparticle (BZI 3 nano) which can be potential carrier to deliver hydrophilic drugs to target colorectum.

Benzimidazole derivative nanoparticle explored to benefit modern pharmacotherapy and drug discovery. Since only limited scientific studies have been carried out so far, a great potential exists to probe the potential therapeutic benefit from the benzimidazole derivatives nanoparticles used in cancer therapy. For reaping such benefit, improving future synthetic research in the cancer therapy is essential.

\section{ACKNOWLEDGEMENT}

The authors are thankful to the Department of pharmaceutical chemistry of RVS college of Pharmaceutical Sciences, Sulur, Tamilnadu, India for providing laboratory facility to carry out the work.

\section{CONFLICT OF INTEREST}

All authors have none to declare.

\section{ABBREVIATIONS}

ACF: Aberrant Crypt Foci, EDTA: Ethylene Diamine Tetra Acetic Acid, DMH: 1, 2 Dimethylhydrazine, BZI 3: Benzimidazole derivative, BZI 3 nano: Benzimidazole derivative nanoparticle, FU: 5-Fluorouracil.

\section{SUMMARY}

Nanotechnology, which are being engineered for the targeted delivery of anticancer drugs. Traditional anticancer agents have a drawback that they need an active transport mechanism to penetrate the cells. This is a major drawback to reach adequate concentration of drug inside the cells. To overcome this disadvantage and developed a novel anticancer agent as benzimidazole derivative nano particle, that could be selective and good target for drug discovery. Used nanotechnology for construct the synthesized benzimidazole derivative as nano particle for provide the customizable, targeted drug delivery vehicles capable of ferrying large 
doses of chemotherapeutic agents or therapeutic genes into malignant cells while sparing healthy cells. in-vivo anticancer activity of novel benzimidazole nanoparticle (BZI 3 nano) results revealed that the presence substituted benzimidazole derivative nanoparticle could have the anticancer potential of the scaffold and selective, good target for drug discovery, which can be regarded as promising anticancer potential.

\section{REFERENCES}

1. Eggadi V, Gundamedi S, Bhava S, Sheshagiri B, Revoori SK, et al. Evaluation of Anticancer Activity of Annona muricata in 1, 2-Dimethyl Hydrazine Induced Colon Cancer, World Applied Sciences Journal , 2014;32(3):444-50.

2. Devasena T, Rajasekaran KN, Gunasekaran G, Viswanathan P, Menon VP. Anticarcinogenic effect of bis-1, 7-(2-hydroxyphenyl)- hepta-1,6-diene-3,5-dione a curcumin analog on $\mathrm{DMH}$-induced colon cancer models. Pharmacological. Resarch. 2003;47(2):133-40.

3. Salim El, Abou-Shafey AE, Masoud AA, Elgendy SA. Cancer Chemopreventive Potential of the Egyptian Flaxseed Oil in a Rat Colon Carcinogenesis BioassayImplications for its Mechanism of Action. Asian Pacific Journal of Cancer Prevention. 2011;12(9):2385-92.

4. Tebbutta NC, Cattellb E. Systemic treatment of colorectal cancer, European Journal of Cancer. 2002;38:1000-15.

5. Walia R, MD. Hedaitullah, Naaz SF, lqbal K, et al. Benzimidazole Derivatives An Overview. International Journal of Research in Pharmacy and Chemistry. 2011;1(3):565-74.

6. El AR, Aboul-Enein HY, et al. Benzimidazole Derivatives as Potential Anticancer Agents. Mini Reviews in Medicinal Chemistry. 2013;3(9):399-407.

7. Refaat HM. Synthesis and anticancer activity of some novel 2-subtituted benzimidazole derivatives. Eur J Med Chem. 2010;45(7):2949-56.

8. Pourgholami MH, Woon L, Almajd R, Akhter J. In vitro and in vivo suppression of growth of hepatocellular carcinoma cells by albendazole. Cancer Lett. 2001; 165(1):43-9.

9. Rajendran SS, Geetha G, Venkatanarayanan R, Santhi N. Formulation, character ization and Anti-cancer activity of novel Benzimidazole derivative nanoparticles. IJRPC. 2018;8(1):96-104.

10. Rajendran SS, Geetha G, Venkatanarayanan R, Santhi N. Synthesis, Characterization And In-vitro Anticancer Evaluation Of Novel Benzo[D]Imidazole Derivatives. IJPSR. 2017;8(7):3014-24.
11. Pathakota R. Pharmacological evaluation of aegle marmelos for anticolon cancer activity in 1, 2 dimethylhydrazine induced colon cancer in rats. World journal of pharmacy and pharmaceutical sciences. 2016;5(6):1381-99.

12. Perse $M$, Cerar A. The dimethylhydrazine induced colorectal tumours in rat experimental colorectal carcinogenesis. Radiol Oncol. 2005; 39(1): 61-70.

13. Patil AB, Jadhav AS. In-vivo and in-vitro models on colon cancer. IJPBS 2013:3(1):24-31.

14. Lorke D. A new approach to practical acute toxicity testing. Arch. Toxicol. 1983;54(4):275-87.

15. OECD/OCDC, OECD "Guidelines for Testing of Chemicals" Revised Draft Guidelines 423; Acute Oral Toxicity.

16. Rodrigues MA, Silva LA, Saladori DM, Camargo JL. Aberrant crypt foci and colon cancer comparison between a short-and medium-term bioassay for colon carcinogenesis using dimethylhydrazine in wistar rats. Braz J Med Biol Res. 2002;35(3):351-5

17. Mecklin JP, Svendsen LB, Peltomäki P, Vasen HF. Hereditary nonpolyposis colorectal cancer. Scandinavian Journal of Gastroenterology. 1994;29(8):673-7.

18. Aranganathan S, Selvam P, Sangeetha N, Nalini N. Modulatory efficacy of hesperetin (citrus flavanone) on xenobiotic-metabolizing enzymes during 1, 2-dimethylhydrazine-induced colon carcinogenesis. Chem. Biol Interact. 2009;180(2):254-61.

19. Samaha HS, Kelloff GJ, Steele V, Rao CV, Reddy BS. Modulation of apoptosis by sulindac, curcumin, phenylethyl-3-methylcaffeate and 6-phenylhexyl isothiocyanate: Apoptotic index as a biomarker in colon cancer chemoprevention and promotion. Cancer Res. 1997;57(7):1301-5.

20. Roshni PS, Dhaval M, Ramesh KG. Anti-cancer activity of colon specific osmotic drug delivery of acetone extract of Quercus Infectoria Olivier, Fagaceae in 1, 2-dimethylhydrazine-induced colon cancer in rats. Molecular Cytogenetics, $2014 ; 7(1): 14$.

21. Dias MC, Spinardi AL, Rodrigues MA, De Camargo JL, Teran E, et al. Lack of chemopreventive effects of ginger on colon carcinogenesis induced by 1, 2-dimethylhydrazine in rats. Food and chem- toxicol. 2006;44(6):877-84.

22. Alrawi SJ, Schiff M, Carroll RE, Gibbs DM, et al. Aberrant crypt foci. Anticancer Res. 2006;26(1A):107-19.

Article History: Submission Date : 05-03-2018; Revised Date : 09-04-2018; Acceptance Date : 04-05-2018.

Cite this article: Rajendran SS, Geetha G, Venkatanarayanan R, Santhi N. Amelioration of 1, 2 Dimethylhydrazine Induced Tumor Promotion Response by Novel Benzimidazole Derivative Nanoparticle in Wistar Rats. J Young Pharm. 2018;10(3):292-8. 